\title{
ELECTROLYSIS-BASED ON-CHIP DISPENSING SYSTEM FOR ESI-MS
}

\author{
Jun Xie*, Qing He*, Yu-Chong Tai*, Jun Liu** and Terry Lee** \\ * Caltech Micromachining Lab, California Institute of Technology, Pasadena, CA 91125, USA \\ ** Beckman Research Institute of the City of Hope, Duarte, CA 91010, USA
}

\begin{abstract}
We report here an integrated on-chip sample dispensing system for Electrospray Ionization-Mass Spectrometry (ESI-MS) applications. The stand-alone chip includes an electrolysis-based micropump, a passive micro mixer and an ESI nozzle. Operation of the chip doesn' $t$ require any external fluidic coupling because the chip is designed to have samples filled, sealed and stored in reservoirs inside the chip before testing. Demonstrated here is a chip with two sample reservoirs and dispensing of the samples is electrically controlled individually. Experimentally, on-chip co-dispensing of two different samples is successfully achieved with a dispensing flow rate about $50 \mathrm{nl} / \mathrm{min}$ and a continuous spray for 2 minutes.
\end{abstract}

\section{INTRODUCTION}

ESI-MS is a powerful tool for studying analytical chemistry or biochemistry. As the technology is pushing for speed and better sensitivity, there are a lot of effort to make stand-alone on-chip microfluidic dispensing systems for ESI-MS because of the advantages of small dead volume, small sample volume, automation, high-throughput, etc. But system integration often involves complex packaging, and in most cases external fluidic coupling is unavoidable. This has become one of the major challenges for miniaturizing dispensing systems To attack this problem, we have designed and fabricated a self-contained chip with many integrated microfluidic components.

Usually the fluid volume and flow rate in onchip microfluidic systems are very small compared to the relatively large external fluidic couplings. For examples, we have made Parylene ESI nozzle [1] and check valves $[1,2]$ that are supported by extemal fluidic couplings with cross sections at least an order of magnitude bigger than those of the on-chip devices. Such fluidic couplings prove to be laborious and low yield. In this paper, we adopt a new scheme that we will fill, seal and store the samples in on-chip reservoirs before use, so no external fluidic coupling is necessary. This completely eliminates the coupling problems. On the chip, an electrolysis injection pump for sample dispensing is integrated with an ESI nozzle. Electrolysis actuation is chosen because of its ease of electrical control, low temperature operation, low power consumption, and high-pressure production capability [3, 4].

One of the important goals for this project is onchip gradient generation. Two reservoirs can be filled with different solvents. Then a gradual concentration change, hence a gradient generation, of a mixed solution from a common outlet can be produced by precise pumping of the two samples with different time-variant rates. This gradient generation is important for highperformance liquid chromatography (HPLC).

\section{DESIGN}

Our devices are designed and fabricated using a multilayer Parylene process, which is flexible and easy to make complex structure. Here, this Parylene surfacemicromachining technology eliminates the need for multichip packaging and external fluidic couplings, while only electrical connections are necessary.

Figure 1 shows the overall operation principle and design for the on-chip dispensing system. Sample is filled and sealed inside an on-chip reservoir before testing. To dispense the sample, an electrolysis voltage is applied between two electrodes. Pneumatic pressure generated by electrolysis drives the liquid out of the reservoir and into the nozzle. The sample is then fed into MS by ESI.

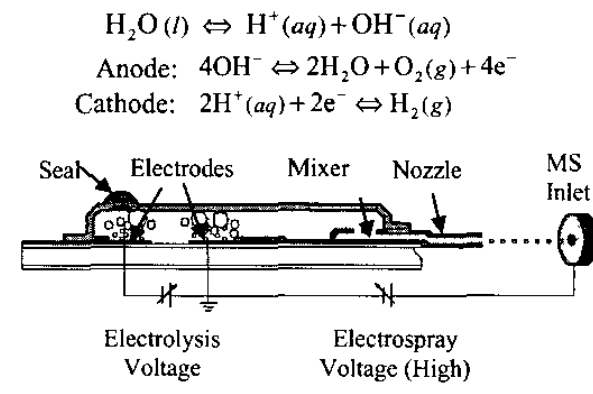

Figure 1. The design and operation of the integrated on-chip dispensing system.

\section{Reservoir}

For conventional desktop ESI-MS instrument that we use, the typical flow rate ranges from 50 to 100 $\mathrm{nL} / \mathrm{min}$ and the time to get a stable spectrum or chromatograph is on the order of several minutes. Since the height of the reservoir is defined by the thickness of sacrificial photoresist, total volume that can be achieved is defined by the area and photoresist height. For convenience, our reservoir dimensions are $1.8 \mathrm{~mm} \times 4$ $\mathrm{mm} \times 15 \mu \mathrm{m}$. The total volume is then $108 \mathrm{~nL}$ and it can sustain a $2-$ minute spray at $50 \mathrm{~nL} / \mathrm{min}$.

The designed reservoir membrane can deflect under the pneumatic pressure generated by the electrolysis. The flexible membrane can prevent the pressure from going too high to make flow rate control difficult. Nevertheless, an array of post is placed inside the reservoir to provide some mechanical support. The 
spacing is empirically chosen to be $300 \mu \mathrm{m}$ as a trade-off between flexibility and strength of the membrane. Since the electrolysis pump is designed to be an injection pump, instead of a reciprocating one, $\mathrm{H}_{2} / \mathrm{O}_{2}$ recombination is not desirable. Therefore, gold, instead of platinum, is chosen as the electrolysis electrode material. Finally, the opening for the liquid filling is a $300 \times 300 \mu \mathrm{m}$ square hole at the corner of the reservoir.

\section{Passive Mixer}

Here a passive mixer is designed without adding any complexity in the fabrication process. Basically, it consists of a high-density ( $5 \mu \mathrm{m}$ spacing) post array to enhance the flow in the perpendicular direction of the main flow. The length of the mixer is designed so that diffusion will allow enough mixing before the liquid is dispensed out of the nozzle under the slow operational flow rate.

\section{ESI Nozzle}

The nozzle design is similar to our previous work [1]. In the past, we used $\mathrm{KOH}$ to etch substrate from the backside to leave a $20 \mu \mathrm{m}$ membrane under the nozzle. After all the process before nozzle freeing, $\mathrm{BrF}_{3}$ is used to etch away the silicon membrane and make the nozzle freestanding and overhanging. Here, we have improved the process to create overhanging nozzle. In this run, $\mathrm{BrF}_{3}$ is used to create freestanding nozzle by etching

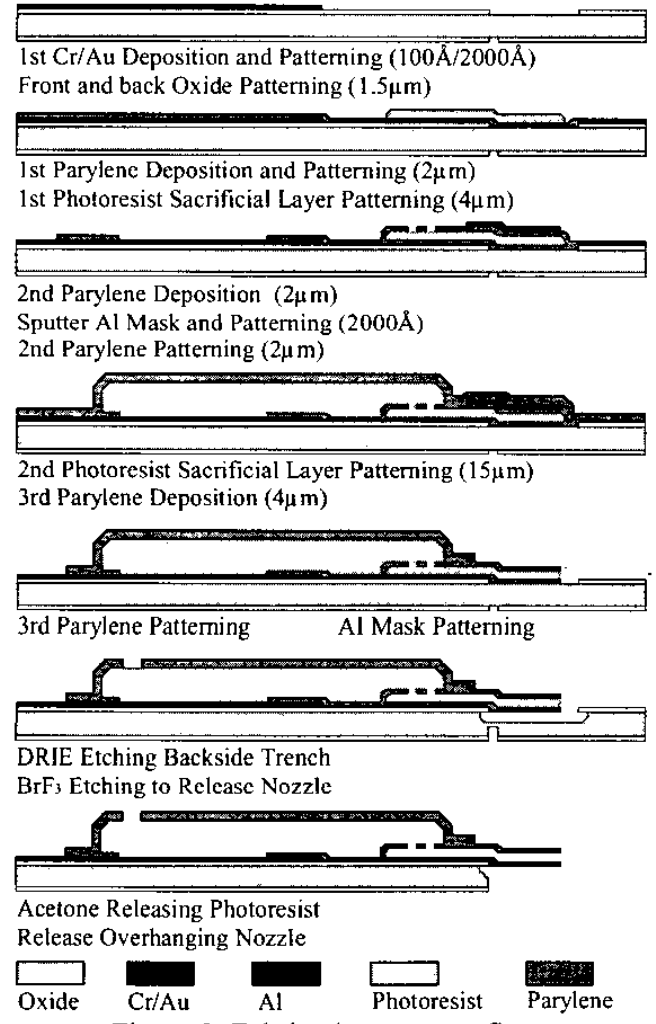

Figure 2. Fabrication process flow. from the front side. It also creates a trench that is later used to break the silicon chip to make the nozzle stick out from the rest of the chip.

\section{FABRICATION}

Fabrication process flow is shown in Figure 2. Process starts with an oxide-coated wafer. $100 \AA 2000 \AA$ $\mathrm{Cr} / \mathrm{Au}$ is evaporated and patterned as the electrodes for electrolysis and ESI. Oxide on both sides is patterned as deep RIE and $\mathrm{BrF}_{3}$ mask. Two $2 \mu \mathrm{m}$ Parylene layers form the channel walls of the micro nozzle and mixer. The 4 $\mu \mathrm{m}$ channel height is defined by the $1^{\text {st }}$ photoresist sacrificial layer. A $2000 \AA$ aluminum is sputtered and patterned. Before the sputtering, Parylene surface is cleaned and roughened in oxygen plasma. This Al layer is the Parylene etching mask to create the sharp ESI nozzle. A $15 \mu \mathrm{m}$ photoresist sacrificial layer and a $4 \mu \mathrm{m}$ Parylene layer define the reservoir. $\mathrm{BrF}_{3}$ dry etching assisted by a DRIE trench creates the overhanging nozzle. Finally, the photoresist sacrificial layers are released by acetone with ultrasonic stirring. Then cleaning continues with methanol and DI water bath.

Figure 3 shows the pictures of the fabricated devices. Figure 3(a) is the micrograph of the ESI nozzle. The overhanging distance from nozzle tip to base is 600 $\mu \mathrm{m}$. This long distance helps to increase the electrical field concentration at the trip and the electrospray. Figure 3 (b) is the micrograph of the whole device with the two reservoirs connected by the micro mixer and the nozzle.

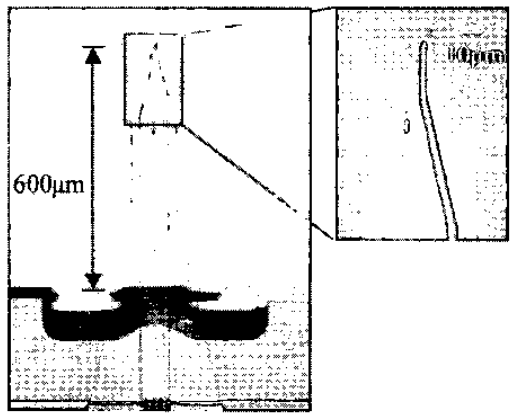

(a)

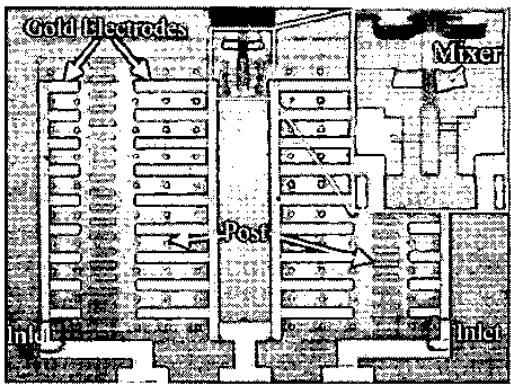

(b)

Figure 3. Micrograph of the fabricated devices. (a) ESI Nozzle (b) Electrolysis Chamber and Micro Mixer. 


\section{TESTING AND RESULTS}

\section{Device Cleaning}

During testing, the sample solutions are introduced by a pipette onto the inlet (the filling hole). Then the surface tension of the samples pulls the liquid inside to fill the reservoir. This requires the inner surface of the reservoir to be clean and hydrophilic. In our case, the bottom surface of the reservoir is silicon dioxide and the top surface is Parylene. We found that during sacrificial photoresist releasing, it tends to leave some residues after drying. This makes the reservoir inner surface hydrophobic and hard for water-based liquid to get in. Thus the cleaning of the devices becomes very important.

Normally, chips are submerged in acetone bath to dissolve the photoresist away. The process is diffusion dominated, so for big reservoir it takes 3 to 4 days to complete the photoresist etching. To enhance cleaning, we have combined both physical and chemical methods. The physical method we used here is ultrasonic agitation. With ultrasonic bath, time for acetone or IPA cleaning is greatly reduced to 10-30 minutes. Nevertheless, when overdone, the ultrasonic bath can produce mechanical damage to the structure. If happens, it mainly attacks the Parylene/Parylene and Parylene/substrate interfaces. Throughout our process, plasma cleaning is performed before deposition of Parylene on Parylene and adhesion promoter is used between Parylene and substrate. The testing shows that long time ultrasonic bath ( $>1$ hour) still can delaminate the reservoir structure. Finally, HF and RCA ultrasonic bath are also tested to clean the surface. It turns out that both of them can cause serious delamination at the same interfaces.

\section{Sample Filling}

After cleaning, the filling of the reservoirs becomes rather easy. On average, $90 \%$ of the chamber can be filled without problems. However, it is found that when the first reservoir is already filled, the second reservoir becomes harder to fill, as shown in Figure 4. The right chamber cannot be filled completely after the left chamber is filled. The small arrows point to the water/air interface in the right chamber. While the left chamber is filled, only half of the right reservoir can be filled after a while. It is believed that the reason for the

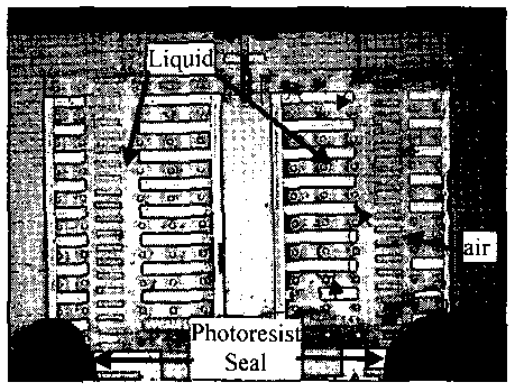

Figure 4. Sample filling of the two connected reservoirs. behavior is that during filling, the air displaced by the liquid needs to vent out. For the chamber first filled, the nozzle and the inlet of second chamber act like the venting opening. But this venting is blocked once the liquid filled the first chamber and the nozzle.

\section{Reservoir Sealing}

After liquid filling, one has to perform sealing before any electrolysis step to prevent liquid leaking out from the filling hole. Permanent sealing using epoxy is not desirable because the chips are intended for multiple recycling uses. In our experiments, we then use photoresist as the sealant shown in Figure 4. It worked for many of our experiments. However, one drawback of using photoresist is that it can be dissolved in many organic solvents such as methanol. Therefore, the photoresist sealant limits the range of organic solvent concentration used in our chip. Also dissolved photoresist will show up as noise in mass spectrum. For water-based sample solutions, photoresist provides an easy and fast way to seal.

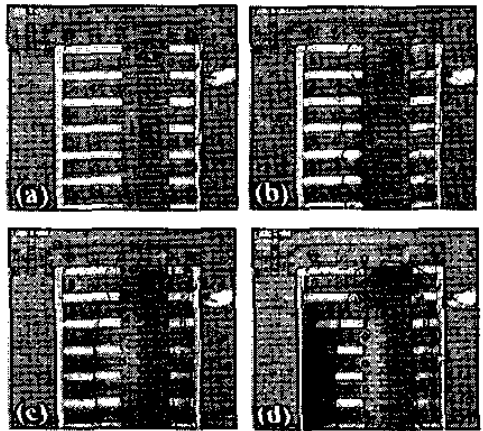

Figure 5. Images from the electrolysis process.

\section{Electrolysis Pump}

After sample is introduced and sealed inside reservoir, an electrolysis voltage $(5-30 \mathrm{~V})$ is applied to the electrodes. Current is typically on the order of 100 to $1000 \mu \mathrm{A}$ depends on the electrolyte properties and the rate of gas production. Bubbles are generated and pressure builds up immediately. Liquid in the reservoir can then be dispensed out of the nozzle by this pneumatic pressure. Figure 5 shows the sequential images from the electrolysis process. In the pictures, shading builds up because the membrane domes up, as in Figure 5(d).

Note that the junction between the reservoir and the mixer is a special feature. It basically connects a tall chamber ( $15 \mu \mathrm{m}$ high) to a small channel ( $4 \mu \mathrm{m}$ high). This geometry difference works as a bubble valve, which requires certain pressure for liquid front to go through the junction due to surface tension. It is found that this, together with the membrane doming effect, results in a delay between the electrolysis bubble generation and the arrival of liquid at the nozzle tip. This is shown by the mass spectrum in Figure 6 that we obtained from the experiments. 


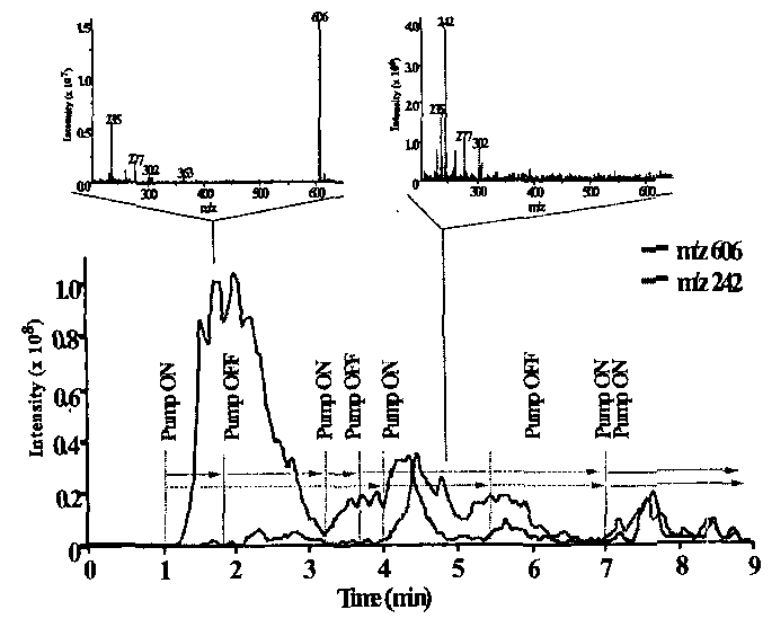

Figure 6. Chromatograph and Mass Spectrum from a two-sample analysis.

As a whole, we demonstrated that liquid injection speed depends on the pressure inside reservoir. Pressure is controlled by the gas generation of electrolysis, which is then controlled by the voltage or current. By adjusting the electrical parameters, the pumping of liquid can be precisely controlled.

\section{Multi-Sample Analysis}

Figure 6 shows the ion chromatograph and representative mass spectrum of a two-sample analysis. Two chambers are filled, respectively, with Tetrabutyl anmonium iodide $(25 \mu \mathrm{M}$, marked as $\mathrm{m} / \mathrm{z} 242)$ and a peptide called leuprolide (30-50 $\mu \mathrm{M}$, marked as $\mathrm{m} / \mathrm{z} 606)$. Both samples are dissolved in 5\% methanol and $5 \%$ acetic acid. Figure 6 clearly shows the delay that is mentioned in the previous section. The flow rate is estimated to be $50 \mathrm{~nL} / \mathrm{min}$ during the first major dispensing in this test.

\section{DISCUSSION}

Although the first generation of the devices is successful, many problems or technology issues still exist. Here we will discuss some of them and suggest possible improvements of the devices.

First of all, the electrolysis happens inside the sample solution. This is undesirable in many applications. In the future, there will be two chambers that are separated by a flexible membrane. Chambers can then be filled with electrolyte (working fluid) and sample solution separately. Pneumatic pressure generated by electrolysis will push the flexible membrane, which then increases the pressure inside sample chamber.

Filling is related to the surface property of the inner surface of the sample reservoir. Photoresist residue makes the filling difficult. To ensure a thorough cleaning, the device has to be able to withstand both physical and chemical cleaning. Adhesion of Parylene needs to be improved since the interface often attacked by cleaning.
Silicon surface roughening by $\mathrm{BrF}_{3}$ has been used and proved to be effective. So our next generation device will incorporate this adhesion enhancement into the process. Since venting is found to be important during filling, structure design can include venting structure to faciliate liquid filling. Although photoresist provides a good seal in some applications, other sealing mechanism must be explored, such as PDMS gasket, thermal bonding between Parylene layers, or self-sealing that exploites the stiction during drying.

\section{CONCLUSION}

An integrated on-chip sample dispensing system for Electrospray Ionization-Mass Spectrometry (ESI-MS) has been successfully developed. The stand-alone system simplifies the experimental process since it doesn' $t$ need any external fluidic coupling. Sample dispensing is electrically controlled. On-chip multi-sample dispensing has been demonstrated. Technical issues associated with components of the system and steps of the testing process are addressed. Many possible improvements for future work are also discussed.

\section{ACKNOWLEDGEMENT}

This work is supported in part by NSF(Grant No. EEC-9402726) and NIH (Grant No. 5R01 RR06217-10). The authors would like to thank Mr. Trevor Roper for the assistance with fabrication.

\section{REFERENCES}

[1] X.Q. Wang, A. Desai, Y.-C. Tai, L. Licklider, T.D. Lee, "Polymer-Based Electrospray Chips for Mass Spectrometry", The 12th IEEE International Conference on Micro Electro Mechanical Systems (MEMS'99), Orlando, Florida, 1999, pp. 523-528

[2] J. Xie, X. Yang, X.Q. Wang and Y.C. Tai, "Surface Micromachined Leakage Proof Parylene Check Valve", The 14th IEEE International Conference on Micro Electro Mechanical Systems (MEMS' 01), Interlaken, Switzerland, 2001, pp.539-542

[3] C.R. Neagu, J.G.E. Gardeniers, M. Elwenspoek, J.J. Kelly, "An Electrochemical Microactuator: Principle and First Results", Journal of MEMS, Vol. 5, No. 1, 1996, pp. $2-9$

[4] S. Böhm, B. Timmer, W. Olthuis and P. Bergveld., "A closed-Loop Controlled Electrochemically Actuated Micro-Dosing System", Journal of Micromechchanics and Microengineering, Vol. 10, No. 4, 2000, pp.498-504 